\title{
A STUDY ON TUMOR-INFILTRATING LYMPHOCYTES IN DIFFERENT SUBTYPES OF BREAST CANCER
}

\author{
Polina D. Dimitrova, \\ Savelina L. Popovska, \\ Ivan N. Ivanov \\ Department of Pathology, \\ Medical University - Pleven
}

\author{
Corresponding Author: \\ Polina D. Dimitrova \\ Department of Pathology, \\ Medical University - Pleven \\ 1, St. Kl. Ohridski Str. \\ Pleven, 5800 \\ Bulgaria \\ e-mail:poldamdim@abv.bg \\ Received: January 15, 2021 \\ Revision received: January 26, 2021 \\ Accepted: April 22, 2021
}

\section{Summary}

The study aimed to investigate immune cell infiltration in different subtypes of breast cancer (BC). Retrospectively were selected 100 patients with primary $\mathrm{BC}$, grouped into four molecular surrogate subtypes (Luminal A and Luminal B-like, HER2-positive and triple-negative - TN), determined by immunohistochemistry (IHC). In each patient, a percentage of stromal tumor-infiltrating lymphocytes (TILs) was determined by hematoxylin-eosin staining. IHC was performed using primary antibodies CD3, CD4, CD8, CD20, and FOXP3. Immunophenotyped lymphocytes were counted (separately intratumoral and stromal) and semiquantitatively graded. In the studied tumors, $10 \%$ were defined as lymphocyte-predominant BC. A high count of intratumoral and stromal TILs subsets was found mainly in TN and HER2-positive BC. The stroma is the preferred localization for immune cells in all four BC subtypes. CD3+ $\mathrm{T}$ predominates over $\mathrm{CD} 20+\mathrm{B}$ lymphocytes, with $\mathrm{CD} 8+$ $\mathrm{T}$ cytotoxic and FoxP3 $+\mathrm{T}$ regulatory cells dominating $\mathrm{T}$ subtypes. HER 2 and $\mathrm{TN}$ are more immunogenic than Luminal A and Luminal B - like subtypes of BC. The T-cells' immune response was predominant in the studied cases of $\mathrm{BC}$, with a predominance of CD8+ Tc and Foxp3+ Treg cells located mainly in the stroma.

Keywords: breast cancer, tumor-infiltrating lymphocytes, immunohistochemistry

\section{Introduction}

The immune system (IS) is responsible for maintaining tissue homeostasis through coordinated activation of innate and acquired immune mechanisms. Neoplastic processes are characterized by tissue and cellular structural and functional changes that induce an immune response (IR) [1]. Tumor-infiltrating lymphocytes (TILs) are local IR directed against tumor growth and progression and are considered an independent favorable prognostic indicator in many malignancies $[2,3]$.

Despite IR, many cases of breast cancer (BC) progress, which calls into question the immune cell infiltrate's adequate function into the tumor microenvironment in this type of neoplasm [2]. The existence of insufficiently effective antitumor immune activity suitable for immune modulation may be 
suspected [4].

The role of immunosuppression in the evolution of cancer is still being studied. Many immunosuppressive factors are present in the tumor, whose presence could be a normal host response to antitumor immunity regulation and/or the presence of tumor-induced immunosuppression $[4,5]$.

Quantification of TILs in BC itself does not reflect the dynamics and functionality of the tumor microenvironment. A better understanding of their role requires determining the lymphocyte phenotype and comparing the different TILs subtypes to BC subtypes' clinical and pathological characteristics.

\section{Materials and methods}

\section{Patients}

Retrospectively, 100 patients with primary $\mathrm{BC}$ were selected, divided into four groups of 25 patients each, according to the molecular surrogate subtypes: Luminal A, Luminal B-like, HER2-positive, and triple-negative - TN.

The patients studied were randomly selected from archival lists of the Department of General and Clinical Pathology at the Georgi Stranski University Hospital -Pleven, based on selecting tumors from archival materials. The materials contained a sufficient amount of tissue samples, and research would in no way threaten the samples with damage and exhaustion.

By the time the patients were selected, we had examined 290 cases of $\mathrm{BC}$, diagnosed in the period 31.12.2014-06.01.2011. All the patients selected for the study had no evidence of inflammatory diseases or other conditions associated with an inflammatory reaction in the breast. The cases in which preoperative antitumor therapy had been given, those for which only specimens from core biopsies were available or archival materials were missing, were excluded.

Hematoxylin and eosin (HE), ER, PR, HER 2, and Ki67 staining archival slides of the patients were examined. One histological slide was selected for each patient to assess the percentage of stromal TILs (TILs \%) in HE staining. We prepared slides to evaluate the TILs subtype populations in staining with immunohistochemistry (IHC) from its corresponding paraffin block.
The ethics commission at the Medical University - Pleven approved the projects. All patient data were summarized and coded, and tissue samples were used without identifying the cases studied.

Demographic data and the clinicopathological factors studied (the type of surgery, tumor stage, histological degree of differentiation (grade - G), histological variant, lymph node (LN) status, lymphovascular invasion (LVI), values for ER, PR, HER2, and Ki-67) were recorded on a questionnaire specially designed for the study.

\section{Histological examination (following the recommendations current at the time of the study)}

The cases of $\mathrm{BC}$ were subtyped according to the WHO histological classification [6]. The Nottingham grading system (modified Elston \& Ellis scheme, 1991) was used to grade the invasive carcinomas [7]. The tumors were staged according to the 7 th revision of the TNM classification of the American Joint Committee on Cancer (AJCC) and the Union for International Cancer Control (UICC) from 2010 [8]. Microscopic evaluation of ER, PR, and HER-2 / neu was performed according to the recommendations of the American Society of Clinical Oncology / College of American Pathologists Guidelines (ASCO/CAP guidelines) $[9,10]$. The interpretation of the IHC results for Ki67 was performed according to the recommendations of the Working Group on BC [11].

The clinically pathological surrogate subtypes of $\mathrm{BC}$ were defined based on the IHC results for ER, PR, HER-2 / neu, and Ki67, according to the St Gallen international expert consensus for primary treatment of early BC 2013 [12].

The microscopic evaluation of TILs in routinely stained (HE) tissue sections was based on the recommendations of the International TILs Working Group 2014, with computerassisted scoring of the percentage of stromal TILs (TILs \%) [1].

\section{Immunohistochemical examination}

From formalin fixed-paraffin embedded tissue specimens, tissue sections were made, with a thickness of 3-4 $\mu \mathrm{m}$, placed on 7109-A Silanized Microscope slides. 
For the IHC study, a visualization system EnVision тм FLEX, High pH (DAKO), AutostainerLink 48 technique (DAKO), and the following primary antibodies were used: CD3 (Polyclonal, Rb, RTU, Dako, Glostrup, Denmark), CD4 (clone 4B12, Mo, RTU, Dako, Glostrup, Denmark), CD8 (clone C8 / 144B, Mo, RTU, Dako, Glostrup, Denmark), CD20 (clone L26, Mo, RTU, Dako, Glostrup, Denmark) and FoxP3 (clone 236A / E7, Mo, 1:100, Bioscience, San Diego, CA USA).

A computer-assisted scoring of positive IHC stained cells for CD3, CD4, CD8, CD20, and FOXP3 was performed independently by two pathologists who had no information about the clinical and pathological data of the studied cases.

The immunophenotyped intratumoral and stromal lymphocytes were counted separately and then were graded semi-quantitatively.

$\mathrm{CD} 3+, \mathrm{CD} 4+, \mathrm{CD} 8+$ and CD20 + TILs were scored in the tumor and stroma of five randomly selected fields, at high magnification (high power fields - HPF), x400. Results with up to $25 \mathrm{IHC}$-positive cells were considered as low, and more than 25 positive cells were considered as high TILs values.

FoxP3-positive lymphocytes were also scored semi-quantitatively in the tumor and stroma, but in at least ten fields of tumor area, at high magnification. The presence of $<15$ and $\geq$ 15 FoxP3-positive cells was reported as low or high FoxP3 expression, respectively.

\section{Statistical analysis}

The obtained results were summarized and statistically analyzed by software (IBM SPSS Statistics V21).

The frequency of the studied clinicopathological characteristics and the semi-quantitatively graded intratumoral and stromal TILs subtypes was determined using the Frequency Table. Crosstab and Chi-Square Test were used to determine the correlations between BC subtypes (despite the small number of cases studied from each molecular surrogate $\mathrm{BC}$ subtype) and the results for the number and localization of TILs subsets. The same methods were used to search for correlations between the reported results and the clinicopathological characteristics of the individual BC subtypes. The association between TILs subsets in the different molecular subtypes of BC was investigated by Spearman's rho test.

A value of $p<0.05$ was considered statistically significant.

\section{Results}

\section{Data on the studied patients}

Most of the studied patients were over 45 years of age $(91.0 \%)$. Mastectomies accounted for the majority $(76.0 \%)$ of the surgical interventions. Most of the tumors were classified as invasive ductal (no special type - NST) carcinoma $(83.0 \%)$, and the remainder were defined as lobular $(9.0 \%)$ or other particular morphological types of $\mathrm{BC}(8.0 \%)$. Tumors with low and moderate malignancy (G1 and G2) predominated in number $(\mathrm{n}=54)$ compared to those with high grade (G3) $(n=46)$. LVI was found in 24 cases; 41 of the cases were with a negative status of the axillary LN, and 37 - with a positive one. The majority of patients $(52 \%)$ were in the T1T2 tumor stage.

The distribution of TILs subsets, depending on their localization and amount in all the BC cases studied $(\mathrm{n}=100)$, are presented in Table 1 .

The distribution of TILs subsets in the different BC molecular subtypes $(\mathrm{n}=25)$ is shown in Table 2-5.

When the distribution of TILs was studied, we found that in all subtypes of BC, B-lymphocytes $(\mathrm{CD} 20+)$ and T-lymphocytes $(\mathrm{CD} 3+)$ were more common in the stromal than in the intratumoral area, where their concentration was high. The subtypes of T-lymphocytes (CD4 +, CD8 +, and FoxP $3+$ ) and their high concentration were less often found intratumorally than in the stroma in Luminal A and HER2-positive BC. The same relationship was found for T-helpers $(\mathrm{CD} 4+)$ and T-cytotoxic cells $(\mathrm{CD} 8+)$ in Luminal B and T-helpers $(\mathrm{CD} 4+)$ in TNBC. There was no significant difference in the distribution of TILs (intratumoral versus stromal) as far as semiquantitatively graded regulatory $\mathrm{T}$ lymphocytes $($ FoxP3 + ) in Luminal B and with CD $8+$ and FoxP3 + in TNBC.

It is noteworthy that the high intratumoral concentration of TILs subsets was not observed in any of the studied cases with BC of Luminal A subtype (see Table 2). High intratumoral 
Table 1. Results for the amount and localization of TILs in the studied tumors $(\mathrm{n}=100)$

\begin{tabular}{|c|c|c|c|c|c|c|}
\hline Intratumoral & $n=100(\%)$ & Stromal & $n=100(\%)$ & $\chi^{2}$ & df & $\mathbf{p}$ \\
\hline CD3 & & & & 58.94 & 2 & $<0.0001$ \\
\hline 0 & $10(10.0)$ & & $2(2.0)$ & & & \\
\hline Low & $76(76.0)$ & Low & $31(31.0)$ & & & \\
\hline High & $14(14.0)$ & High & $67(67.0)$ & & & \\
\hline CD4 & & & & 69.55 & 2 & $<0.0001$ \\
\hline 0 & $65(65.0)$ & & $9(9.0)$ & & & \\
\hline Low & $35(25.0)$ & Low & $84(84.0)$ & & & \\
\hline High & 0 & High & $7(7.0)$ & & & \\
\hline CD8 & & & & 36.63 & 2 & $<0.0001$ \\
\hline 0 & $20(20.0)$ & & $1(1.0)$ & & & \\
\hline Low & $72(72.0)$ & Low & $62(62.0)$ & & & \\
\hline High & $8(8.0)$ & High & $37(37.0)$ & & & \\
\hline FoxP3 & & & & 28.49 & 2 & $<0.0001$ \\
\hline 0 & $25(25.0)$ & 0 & $5(5.0)$ & & & \\
\hline Low & $70(70.0)$ & Low & $68(68.0)$ & & & \\
\hline High & $5(5.0)$ & high & $27(27.0)$ & & & \\
\hline CD20 & & & & 52.99 & 2 & $<0.0001$ \\
\hline 0 & $41(41.0)$ & 0 & $3(3.0)$ & & & \\
\hline Low & $59(59.0)$ & Low & $80(80.0)$ & & & \\
\hline High & 0 & High & $17(17.0)$ & & & \\
\hline \multicolumn{7}{|l|}{ TIL (\%) } \\
\hline$\leq 50 \%$ & & & $90(90.0)$ & & & \\
\hline$>50 \%$ & & & $10(10.0)$ & & & \\
\hline
\end{tabular}

Table 2. Results for the amount and localization of TILs in Luminal A subtype of BC $(\mathrm{n}=25)$

\begin{tabular}{|c|c|c|c|c|c|c|}
\hline Intratumoral & $n=25(\%)$ & Stromal & $n=25(\%)$ & $\chi^{2}$ & df & $\bar{p}$ \\
\hline CD3 & & & & 21.12 & 2 & $<0.0001$ \\
\hline 0 & $4(16.0)$ & & & & & \\
\hline Low & $21(84.0)$ & Low & $11(44.0)$ & & & \\
\hline High & $0(0)$ & High & $14(56.0)$ & & & \\
\hline CD4 & & & & 24.74 & 2 & $<0.0001$ \\
\hline 0 & $18(72.0)$ & & $1(4.0)$ & & & \\
\hline Low & $7(28.0)$ & Low & $23(92.0)$ & & & \\
\hline High & 0 & High & $1(4.0)$ & & & \\
\hline CD8 & & & & 13.03 & 2 & 0.0015 \\
\hline 0 & $6(24.0)$ & & $0(0)$ & & & \\
\hline Low & $19(76.0)$ & Low & $18(72.0)$ & & & \\
\hline High & $0(0)$ & High & $7(28.0)$ & & & \\
\hline FoxP3 & & & & 17.06 & 2 & 0.0002 \\
\hline 0 & $11(44.0)$ & 0 & $0(0)$ & & & \\
\hline Low & $14(56.0)$ & Low & $20(80.0)$ & & & \\
\hline High & $0(0)$ & High & $5(20.0)$ & & & \\
\hline CD20 & & & & 16.29 & 2 & 0.0003 \\
\hline 0 & $13(52.0)$ & 0 & $1(4.0)$ & & & \\
\hline Low & $12(48.0)$ & Low & $20(80.0)$ & & & \\
\hline High & $0(0)$ & High & $4(16.0)$ & & & \\
\hline \multicolumn{7}{|l|}{ TIL (\%) } \\
\hline$\leq 50 \%$ & & & $25(100.0)$ & & & \\
\hline$>50 \%$ & & & $0(0)$ & & & \\
\hline
\end{tabular}


Table 3. Results for the amount and localization of TILs in Luminal B subtype of BC $(\mathrm{n}=25)$

\begin{tabular}{|c|c|c|c|c|c|c|}
\hline Intratumoral & $n=25(\%)$ & Stromal & $n=25(\%)$ & $\chi^{2}$ & df & p \\
\hline CD3 & & & & 18.79 & 2 & $<0.0001$ \\
\hline 0 & $2(8.0)$ & & & & & \\
\hline Low & $20(80.0)$ & Low & $7(28.0)$ & & & \\
\hline High & $3(12.0)$ & High & $18(72.0)$ & & & \\
\hline CD4 & & & & 12.52 & 2 & 0.0019 \\
\hline 0 & $13(52.0)$ & & $2(8.0)$ & & & \\
\hline Low & $12(48.0)$ & Low & $21(84.0)$ & & & \\
\hline High & & High & $2(8.0)$ & & & \\
\hline CD8 & & & & 10.48 & 2 & 0.0053 \\
\hline 0 & $6(24.0)$ & & $0(0)$ & & & \\
\hline Low & $17(68.0)$ & Low & $16(64.0)$ & & & \\
\hline High & $2(8.0)$ & High & $9(36.0)$ & & & \\
\hline FoxP3 & & & & 5.69 & 2 & 0.0581 \\
\hline 0 & $3(12.0)$ & 0 & $0(0)$ & & & \\
\hline Low & $21(84.0)$ & Low & $20(80.0)$ & & & \\
\hline High & $1(4.0)$ & High & $5(20.0)$ & & & \\
\hline CD20 & & & & 14.19 & 2 & 0.0008 \\
\hline 0 & $12(48.0)$ & 0 & $1(4.0)$ & & & \\
\hline Low & $13(52.0)$ & Low & $21(84.0)$ & & & \\
\hline High & $0(0)$ & High & $3(12.0)$ & & & \\
\hline \multicolumn{7}{|l|}{ TIL (\%) } \\
\hline$\leq 50 \%$ & & & $24(96.0)$ & & & \\
\hline$>50 \%$ & & & $1(4.0)$ & & & \\
\hline
\end{tabular}

Table 4. Results for the amount and localization of TILs in HER2-positive subtype of BC $(\mathrm{n}=25)$

\begin{tabular}{|c|c|c|c|c|c|c|}
\hline Intratumoral & $n=25(\%)$ & Stromal & $n=25(\%)$ & $\chi^{2}$ & df & $\mathbf{p}$ \\
\hline CD3 & & & & 16.45 & 2 & 0.0003 \\
\hline 0 & $1(4.0)$ & & $1(4.0)$ & & & \\
\hline Low & $20(80.0)$ & Low & $6(24.0)$ & & & \\
\hline High & $4(16.0)$ & High & $18(72.0)$ & & & \\
\hline CD4 & & & & 21.17 & 2 & $<0.0001$ \\
\hline 0 & $19(76.0)$ & & $3(12.0)$ & & & \\
\hline Low & $6(24.0)$ & Low & $20(80.0)$ & & & \\
\hline High & $0(0)$ & High & $2(8.0)$ & & & \\
\hline CD8 & & & & 10.29 & 2 & 0.0058 \\
\hline 0 & $3(12.0)$ & & $0(0)$ & & & \\
\hline Low & $20(80.0)$ & Low & $14(56.0)$ & & & \\
\hline High & $2(8.0)$ & High & $11(44.0)$ & & & \\
\hline FoxP3 & & & & 9.52 & 2 & 0.0086 \\
\hline 0 & $4(16.0)$ & 0 & $2(8.0)$ & & & \\
\hline Low & $20(80.0)$ & Low & $13(52.0)$ & & & \\
\hline High & $1(4.0)$ & High & $10(40.0)$ & & & \\
\hline CD20 & & & & 10.10 & 2 & 0.0064 \\
\hline 0 & $6(24.0)$ & 0 & $0(0)$ & & & \\
\hline Low & $19(76.0)$ & Low & $21(84.0)$ & & & \\
\hline High & $0(0)$ & High & $4(16.0)$ & & & \\
\hline \multicolumn{7}{|l|}{ TIL (\%) } \\
\hline$\leq 50 \%$ & & & $20(80.0)$ & & & \\
\hline$>50 \%$ & & & $5(20.0)$ & & & \\
\hline
\end{tabular}


— Dimitrova P., et al. A study on tumor-infiltrating lymphocytes in different subtypes ...

Table 5. Results for the amount and localization of TILs in TN subtype of BC $(n=25)$

\begin{tabular}{|c|c|c|c|c|c|c|}
\hline Intratumoral & $n=25(\%)$ & Stromal & $n=25(\%)$ & $x^{2}$ & df & $\mathbf{p}$ \\
\hline CD3 & & & & 8.08 & 2 & 0.0176 \\
\hline 0 & $3(12.0)$ & & $1(4.0)$ & & & \\
\hline Low & $15(60.0)$ & Low & $7(28.0)$ & & & \\
\hline High & $7(28.0)$ & High & $17(68.0)$ & & & \\
\hline $\mathrm{CD} 4$ & & & & 13.33 & 2 & 0.0013 \\
\hline 0 & $15(60.0)$ & & $3(12.0)$ & & & \\
\hline Low & $10(40.0)$ & Low & $20(80.0)$ & & & \\
\hline High & $0(0)$ & High & $2(8.0)$ & & & \\
\hline CD8 & & & & 5.37 & 2 & 0.0682 \\
\hline 0 & $5(20.0)$ & & $1(4.0)$ & & & \\
\hline Low & $16(64.0)$ & Low & $14(56.0)$ & & & \\
\hline High & $4(16.0)$ & High & $10(40.0)$ & & & \\
\hline FoxP3 & & & & 2.20 & 2 & 0.2019 \\
\hline 0 & $7(28.0)$ & 0 & $3(12.0)$ & & & \\
\hline Low & $15(60.0)$ & Low & $15(60.0)$ & & & \\
\hline High & $3(12.0)$ & High & $7(28.0)$ & & & \\
\hline CD20 & & & & 13.64 & 2 & 0.0011 \\
\hline 0 & $10(40.0)$ & 0 & $1(4.0)$ & & & \\
\hline Low & $15(60.0)$ & Low & $18(72.0)$ & & & \\
\hline High & $0(0)$ & High & $6(24.0)$ & & & \\
\hline \multicolumn{7}{|l|}{ TIL (\%) } \\
\hline $\begin{array}{l}\leq 50 \% \\
>50 \%\end{array}$ & & & $\begin{array}{l}21(84.0) \\
4(16.0)\end{array}$ & & & \\
\hline
\end{tabular}

concentrations were found in a small number of cases in Luminal B and HER2 - positive BC (see Table 3 and 4). Of all the BC subtypes, the high concentration of TILs intratumorally was mostly established in the TN subtype (see Table 5).

High stromal concentrations of TILs subspecies were found in most BC cases with TN and HER2-positive, compared with those with Luminal A and Luminal B (see Table 2-5).

\section{Predominant subsets of lymphocytes in the subtypes of $B C$}

In Luminal A subtype BC, CD3 + T-lymphocytes predominated over CD20 + B-lymphocytes intratumorally $(\chi 2=5.70 ; \mathrm{Df}=1 ; \mathrm{p}=0.0196)$ and stromally $(\chi 2=9.17$; Df $=2 ; p=0.0102)$. CD3 + T-lymphocytes predominated over CD20 + B-lymphocytes intratumorally and in Luminal B subtype $(\chi 2=11.63 ; \mathrm{Df}=2 ; \mathrm{p}=0.0030)$; HER2- positive $(\chi 2=7.60 ; \mathrm{Df}=2 ; \mathrm{p}=0.0224)$ and $\mathrm{TN}(\chi 2=10.77 ; \mathrm{Df}=2 ; \mathrm{p}=0.0046) \mathrm{BC}$.

Like in Luminal A BC, CD3 + T lymphocytes predominated over CD20 + B lymphocytes in the tumor stroma in other surrogate types of $\mathrm{BC}$ : Luminal B type $(\chi 2=18.71 ; \mathrm{Df}=2 ; \mathrm{p}=0.0001)$; HER2 positive $(\chi 2=18.24 ; \mathrm{Df}=2 ; \mathrm{p}=0.0001)$ and TN $(\chi 2=10.10 ; \mathrm{Df}=2 ; \mathrm{p}=0.0064) \mathrm{BC}$.

$\mathrm{CD} 8$ - $\mathrm{T}$ cytotoxic $(\mathrm{Tc})$ and FoxP3-T regulatory cells (Treg) dominated among the T-subtypes, and the domination had a statistically significant value in Luminal B, HER2, and TN subtypes of BC - Table 6.

B-cell CD20 + humoral IR was not a prominent feature in $\mathrm{BC}$ and is found mainly in the HER2-positive subtype, while the highest concentration of B-lymphocytes was found in the TN BC subtype (see Table 2 - 5).

\section{Correlations between the subtypes of lymphocyte infiltrate}

CD8 + Tc and Foxp3 + Treg cells are more commonly seen together intratumorally in Luminal B, HER2-positive, and TN BC subtypes, and stromally in the Luminal B and TN BC ones.

\section{Immunogenicity of BC subtypes}

Despite the small number of cases studied from each surrogate BC subtype, it was found that a high concentration of intratumoral and stromal lymphocytes was found predominantly in $\mathrm{TN}$, followed by HER2-positive BC - Figures 1 and 2. 
Table 6. Statistically significant correlations between lymphocyte subspecies in different molecular subtypes BC

\begin{tabular}{|c|c|c|c|c|c|c|c|}
\hline \multicolumn{2}{|c|}{ Intratumoral } & \multirow[t]{2}{*}{ BC subtype } & \multirow[t]{2}{*}{$\mathbf{p}$} & \multicolumn{2}{|c|}{ Stromal } & \multirow[t]{2}{*}{ BC subtype } & \multirow[t]{2}{*}{ p } \\
\hline CD3 & CD8 & & & CD3 & CD8 & & \\
\hline & & Luminal B & 0.001 & & & Luminal B & 0.018 \\
\hline & & $\mathrm{TN}$ & 0.002 & & & & \\
\hline \multirow[t]{4}{*}{ CD3 } & FoxP3 & & & CD3 & FoxP3 & & \\
\hline & & Luminal B & 0.008 & & & HER-2 & 0.011 \\
\hline & & HER-2 & 0.035 & & & & \\
\hline & & $\mathrm{TN}$ & 0.010 & & & & \\
\hline \multirow[t]{4}{*}{ CD8 } & FoxP3 & & & CD8 & FoxP3 & & \\
\hline & & Luminal B & 0.002 & & & Luminal B & 0.021 \\
\hline & & HER2 & 0.001 & & & $\mathrm{TN}$ & 0.008 \\
\hline & & $\mathrm{TN}$ & 0.001 & & & & \\
\hline
\end{tabular}
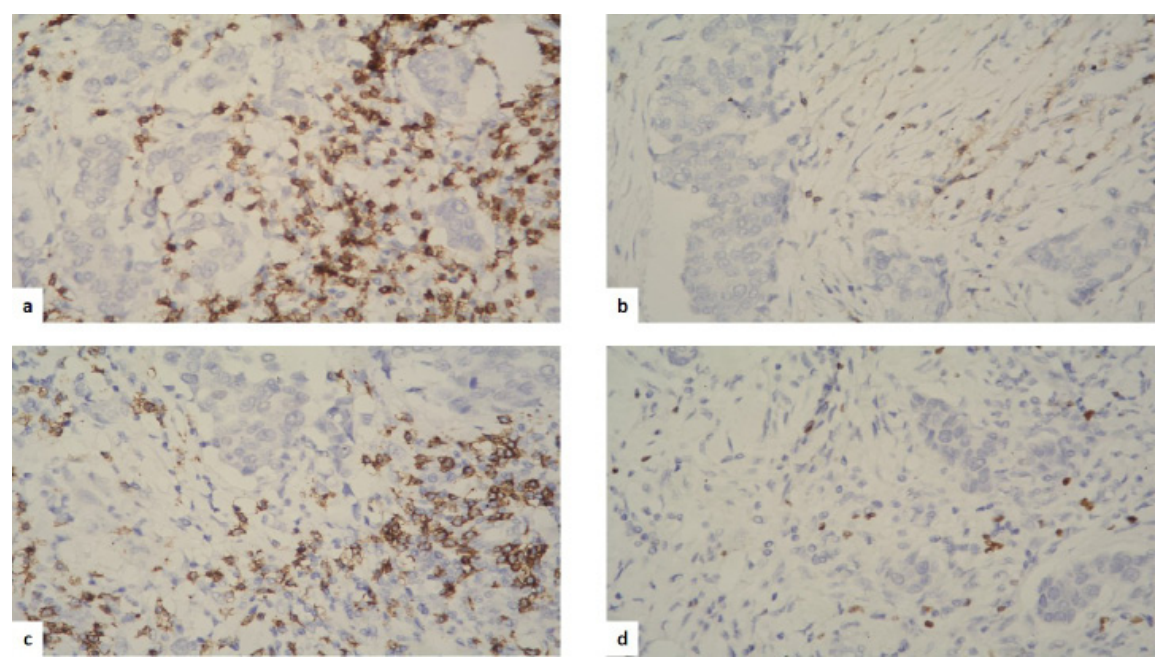

Figure 1. Microscopic evaluation of IHC stained T-lymphocyte subtypes (a - CD3; b - CD4; c - CD8; d- FoxP3) in HER2-positive BC, $\mathrm{x} 400$
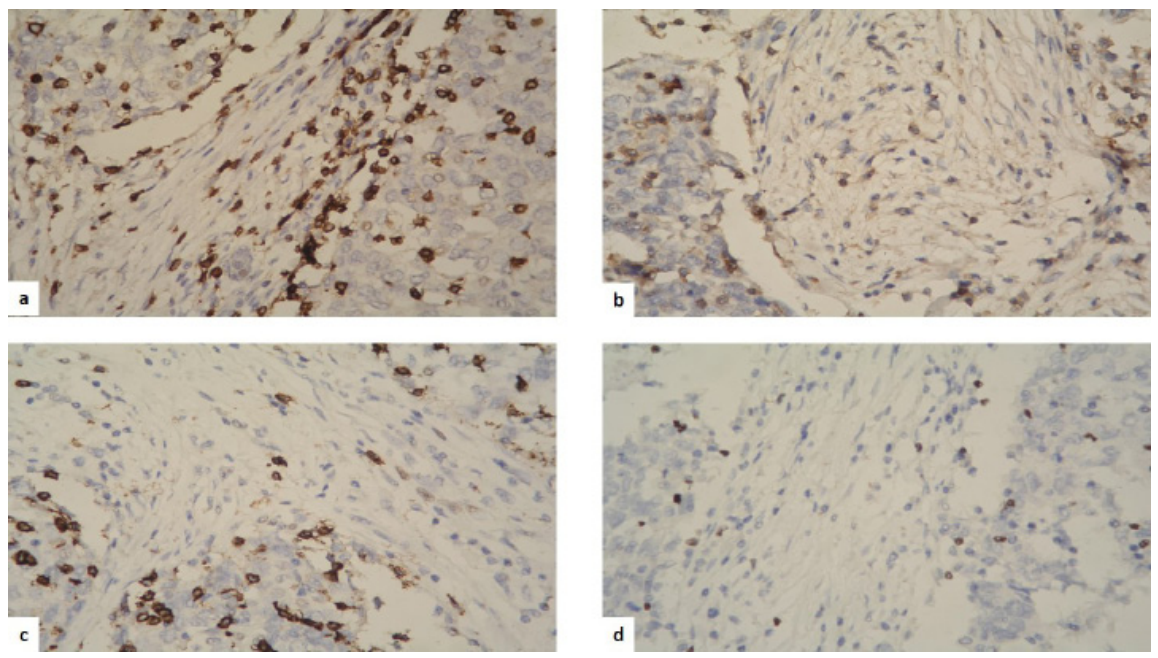

Figure 2. Microscopic evaluation of IHC stained T-lymphocyte subtypes (a - CD3; b - CD4; c - CD8; d- FoxP3) in $\mathrm{TNBC}, \mathrm{x} 400$

\section{BC with TIL> 50\% (Lymphocyte- predominant $B C-L P B C)$}

Of the tumors studied, $10 \%$ were defined as LPBC: HER2-positive $(\mathrm{n}=5), \mathrm{TN}(\mathrm{n}=4)$ and Luminal B $(n=1)$.
Correlations of TIL with the clinical and pathological characteristics see Table 6

A statistically significant association was found between the number of stromal CD8- 
Tc lymphocytes and lymphovascular invasion (LVI) in HER2-positive tumors $(\mathrm{p}=0.014)$. In this latter subtype of BC, $28 \%$ had LVI, of which $85.7 \%$ had a high count of stromal CD8 Tc lymphocytes.

A correlation was found between the number of stromal FoxP3-Treg lymphocytes and the histological grade $(G)(p=0.015)$ in the Luminal B subtype. A high number of stromal FoxP3+ cells was found in $20 \%$ of the Luminal B cases, all of which (100\%) were G3 BC.

No statistically significant correlation was found between the lymphocyte subspecies and any clinico-pathological parameters studied in Luminal A and TN BC.

\section{Discussion}

Most studies have reported that stromal TILs are a better and more reproducible parameter than intratumoral TILs $[1,13,14]$. The main reason for this difference is that intratumoral TILs are presented less frequently, in smaller quantities, and are difficult to identify in routine staining. IHC allows for their easy visualization and enhances the information obtained in the determination of stromal TILs [15-17].

In all four molecular subtypes of $\mathrm{BC}$ we studied, the major subtypes of immune cells involved in cellular and humoral IR (CD3 + Tand CD20 + B-lymphocytes, respectively) were localized mainly in the stroma. The stroma is also a preferred site for T-lymphocytes (helper CD4 + , cytotoxic $\mathrm{CD} 8+$ and regulatory FoxP3 +) in Luminal A and HER2-positive BC subtypes, as well as for CD4 + and CD8 + in Luminal B and CD4 + in TNBC. No significant difference was found between the intratumoral and intrastromal distribution of TILs, concerning semiquantitatively graded regulatory $\mathrm{T}$ lymphocytes $($ FoxP $3+)$ in Luminal B and with $\mathrm{CD} 8+$ and FoxP3 + in TNBC.

There is accumulated evidence supporting the clinical significance of IR in cancer. Data from clinical trials have demonstrated the potential of immunotherapy, and increased survival rates have been reported in patients with urothelial carcinoma [18], lung cancer [19], and others [1]. Immunotherapy for carcinomas is a possibility for effective treatment by overcoming the phenomenon of immunosuppression - an essential factor involved in the process of tumor development and progression of neoplasms. Active immunotherapy aims to modify the amount or the elements comprising the subgroups of lymphocytes and reactivate antitumor IR, thus improving the outcome of the disease [20].

Clarifying the role of IS and modulation of IR promise new therapeutic opportunities for $\mathrm{BC}$, including $\mathrm{TNBC}$, given the lack of targeted molecular treatment in this aggressive subtype of BC [21]. Cytotoxic treatment, such as chemotherapy and radiation therapy, can stimulate and potentiate the body's immune reactivity, inducing a specific response [22] and immunogenic tumor cell death as an end-result $[1,4,17,21,23]$. Improved survival has been reported in patients with metastatic TNBC on immunotherapy and chemotherapy [24]. The clinical significance of the synergistic effect of combining therapy with other treatment regimens is studied [16].

The complex of cells involved in antitumor immunity is an essential functional factor [17]. It varies depending on the type and organ localization of the neoplasm [1]. For evaluating TILs subtypes in BC, the IHC method with the most commonly used markers, such as CD20, CD3, CD4, CD8, and FoxP3, identifying B, T, and T-lymphocyte subtypes is preferable [20,25].

The CD3 antigen is a receptor glycoprotein found in mature $\mathrm{T}$ lymphocytes. The CD4 antigen is a glycoprotein on the surface of helper $\mathrm{T}$ cells $(\mathrm{Th})$, regulatory $\mathrm{T}$ cells, monocytes, and macrophages. The CD8 antigen is also a $\mathrm{T}$-cell receptor glycoprotein [2] expressed in T-cytotoxic (Tc) cells. CD20 is a transmembrane protein in B-cell precursors and mature B cells, which is lost after differentiation into plasma cells [25].

Regulatory $\mathrm{T}$ lymphocytes (Treg) are a specific CD4 + T-cell population that suppresses the activation of other immune cells and maintains systemic immune homeostasis. Treg cells also play an essential role in suppressing tumor-associated antigen-specific immunity. Foxp3 (Forkhead Box Protein 3) - positive lymphocytes are a more noticeable subpopulation of immunosuppressive regulatory $\mathrm{T}$ cells. It has been suggested that FoxP3 levels may be an indicator of tumorigenesis in $\mathrm{BC}$ [5]. There are conflicting data on their clinical significance in patients with this neoplasm [5, 22, 26, 27]. 
Like in other studies [23, 25], we found that T-cell-mediated immunity was a leading factor in the antitumor response in BC. Humoral B-cell immunity was less pronounced. In all the four molecular subtypes we studied, cases with T-cell CD3 expression (intratumoral and stromal) predominated over those with CD20-positive B lymphocytes. There were more cases with a high concentration of CD3 + T-lymphocytes than cases with high CD20 + B-lymphocyte infiltration. Although they were detected in a smaller number of tumors, most cases of B-lymphocyte expression were detected in the HER2-positive BC subtype, while high concentrations of B-lymphocytes were most commonly found in TNBC.

In our study, CD8-Tc and FoxP3-Treg cells were the predominant subtypes of CD3 + T-lymphocytes in all four molecular subtypes of $\mathrm{BC}$, and it was shown that these subtypes of TILs are more commonly seen together. Statistically significant values are presented in Table 6. The large number of cytotoxic CD8-Tc cells found was probably a signal of an active IS attempt to reject the tumor, which had been unsuccessful due to their suppression by FoxP3 + Treg cells or to a suppressive effect of tumor cells specific to individual molecular subspecies BC. According to data from some studies, other TILs subspecies are predominant. In the literature, there are differences in the study design (selection of $\mathrm{BC}$ subtypes, the study of unequal numbers and different markers for lymphocyte infiltrate, use of different TILs detection techniques and methods for immunophenotyping) $[2,25,28]$.

Among the BC subtypes we studied, TN had the most prominent lymphocyte infiltrate, followed by HER2-positive non-luminal BC. In these two subtypes, there was a high concentration of all TILs subsets in more cases as compared to the Luminal A and Luminal B types of BC. The data we obtained corresponds to those found in other studies, according to which these molecular subtypes of $\mathrm{BC}$ are more immunogenic than others $[13,16,17,21,29,30]$.

The term lymphocyte-predominant BC (LPBC) pertains to tumors in which lymphocytes occupy more than $50 \%-60 \%$ of the stromal area in routinely stained tissue sections [1]. In the present study, $10 \%$ of the neoplasms studied were defined as LPBC: HER2-positive $(n=5)$,
TN $(n=4)$, and Luminal B $(n=1)$, and no case of Luminal A was assigned to this category. Similar results were obtained by Ohtani et al., using a cut-off for TILs of 30\% and 50\%. According to them, $8.2 \%$ of invasive $\mathrm{BC}$ are LPBC, with TN ranking first, followed by HER2 - positive, and Luminal B accounted for the smallest percentage. They reported no cases of Luminal A, defined as LPBC [29].

Identification of new reliable and longterm prognostic factors in $\mathrm{BC}$ remains a major unresolved issue [5,16,17,22,26,31]. The classic markers determine the possibility of progression in the first five years after therapy. After this period, their importance for long-term survival decreases, especially in patients with TNBC [5].

The prognostic significance of TILs and TILs subsets remains a long-standing topic of debate $[2,16,17,22,26,27]$. In some studies, the degree of lymphocyte infiltration assessed by routine staining was found to have prognostic value in TN and HER2 $+\mathrm{BC}$, despite the lack of detailed information on immune subpopulations in the infiltrate $[1,13,16,23,32,33]$. Active and persistent antitumor IR potentially identifies more immunogenic tumors. The different numbers and compositions of lymphocytes reflect the unequal cell biology of carcinomas, with different mechanisms for generating immunological memory and the ability to control residual tumors effectively [1]. It is suggested that lymphocyte subtype scoring may contribute to a better prognosis in $\mathrm{BC}[5,14,17,22,26,27]$. Finding a correlation between specific characteristics of TILs with clinical results and proven prognostic indicators is part of the complex process of including a biomarker in routine practice $[3,15,16,25,26,28,31,34]$. Additional studies are needed before introducing changes in daily clinical practice and using TILs and TILs subtypes as a biological prognostic biomarker [13,16,31].

Our results showed that lymphovascular invasion (LVI) was associated with a high concentration of stromal CD8-Tc in the HER2positive subtype and a low histological degree of differentiation (G3) high count of stromal FoxP3Treg in the Luminal B subtype. Therefore, two of the major functional components of tumorassociated IR: cytotoxic CD8 + and regulatory Foxp3 $+\mathrm{T}$ cells agree with adverse prognostic 
factors such as LVI and G3 as found in other studies [6,7,35-37].

\section{Conclusions}

The results obtained for the amount, localization and immune phenotype of TILs in the different subtypes of BC could be used for additional studies to:

- determine their significance as an independent prognostic marker;

- establish how the influence of these parameters can be used for immunomodulation and creation of an effective antitumor immune response;

- study their role as a predictive marker in selecting patients eligible for specific therapeutic regimens.

Clinical follow-up of patients and disease outcomes should be considered so that TILs and TILs subtypes can be used as a safe and longterm prognostic marker. An in-depth study of the association between the inflammatory infiltrate, and the effect of specific therapy is required to determine the predictive value of TILs.

The mechanisms by which immune cells interact with each other and with the tumor cell population remain unclear. Further investigations on lymphocyte markers in a larger number of patients with each type of $\mathrm{BC}$ are necessary.

Determining the amount of different immune cell populations and their localization in the tumor and comparing them with clinical and pathological data in different types of $\mathrm{BC}$ may serve as a basis for an in-depth analysis of the role of lymphocyte infiltration in the tumorigenesis and progression of this neoplasm. Patients with $\mathrm{BC}$ could be given a new treatment option by developing future therapeutic approaches based on specific biomarkers. The immune system is a promising new target for this goal.

\section{Acknowledgements}

The data presented in this article results from the development of a research project №19 / 2015 at Medical University - Pleven and a project BG05M2OP001-1.002-0010, funded by the "Science and education for smart growth" Operational Program and the European Regional Development Fund.

\section{References}

1. Salgado R, Denkert C, Demaria S, Sirtaine N, Klauschen F, Pruneri G, et al. The evaluation of tumor-infiltrating lymphocytes (TILs) in breast cancer: recommendations by an International TILs Working Group 2014. Ann Oncol. 2015;26(2):259-71.

2. Rathore AS, Kumar S, Konwar R, Makker A, Negi MP, Goel MM. CD3+, CD4+ \& CD8+ tumour infiltrating lymphocytes (TILs) are predictors of favourable survival outcome in infiltrating ductal carcinoma of breast. Indian J Med Res. 2014;140(3):361-9.

3. Rathore AS, Goel MM, Makker A, Kumar S, Srivastava AN. Prognostic Impact of CD3 Tumor Infiltrating Lymphocytes in Triplenegative Breast Cancer. Indian Journal of Clinical Practice. 2013; 24(4): 376-80.

4. Loi S. Host antitumor immunity plays a role in the survival of patients with newly diagnosed triple-negative breast cancer. Journal of Clinical Oncology: Official Journal of the American Society of Clinical Oncology. 2014;32(27):2935-7.

5. Lee S, Cho EY, Park YH, Ahn JS, Im YH. Prognostic impact of FOXP3 expression in triple-negative breast cancer. Acta Oncol. 2013;52(1):73-81.

6. Lakhani SR, Ellis IO, Schnitt SJ, Tan PH, van de Vijver MJ. WHO Classification of Tumours of the Breast. 4th Edition. Lyon: IARC Press; 2012. 240p.

7. Elston CW, Ellis IO. Pathological prognostic factors in breast cancer. I. The value of histological grade in breast cancer: experience from a large study with long-term follow-up. Histopathology. 1991;19(5):403-10.

8. 8. Edge SB, Byrd DR, Compton CC, Fritz AG, Greene FL, Trotti A, editors. AJCC cancer staging manual (7th ed). New York, NY: Springer; 2010.

9. Hammond ME, Hayes DF, Dowsett M, Allred DC, Hagerty KL, Badve S, etal.American Society of Clinical Oncology/College Of American Pathologists guideline recommendations for immunohistochemical testing of estrogen and progesterone receptors in breast cancer. J Clin Oncol. 2010;28(16):2784-95.

10. Wolff AC, Hammond ME, Hicks DG, Dowsett M, McShane LM, Allison KH, et al. Recommendations for human epidermal growth factor receptor 2 testing in breast cancer: American Society of Clinical Oncology/ College of American Pathologists clinical 
practice guideline update. J Clin Oncol. 2013;31(31):3997-4013.

11. Dowsett M, Nielsen TO, A'Hern R, Bartlett J, Coombes RC, Cuzick J, et al. International Ki67 in Breast Cancer Working Group. Assessment of Ki67 in breast cancer: recommendations from the International Ki67 in Breast Cancer working group. J Natl Cancer Inst. 2011;103(22):165664.

12. Goldhirsch A, Winer EP, Coates AS, Gelber $\mathrm{RD}$, Piccart-Gebhart $\mathrm{M}$, Thürlimann $\mathrm{B}$, et al. Personalizing the treatment of women with early breast cancer: highlights of the St Gallen International Expert Consensus on the Primary Therapy of Early Breast Cancer 2013. Ann Oncol. 2013;24(9):2206-23.

13. Kos Z, Roblin E, Kim RS, Michiels S, Gallas BD, Chen $\mathrm{W}$, et al. International Immuno-Oncology Biomarker Working Group. Pitfalls in assessing stromal tumor infiltrating lymphocytes (sTILs) in breast cancer. NPJ Breast Cancer. 2020;6:17.

14. Amgad M, Stovgaard ES, Balslev E, Thagaard J, Chen W, Dudgeon S, et al. International Immuno-Oncology Biomarker Working Group. Report on computational assessment of Tumor Infiltrating Lymphocytes from the International Immuno-Oncology Biomarker Working Group. NPJ Breast Cancer. 2020;6:16.

15. Klauschen F, Müller KR, Binder A, Bockmayr M, Hägele $M$, Seegerer $P$, et al. International Immuno-Oncology Biomarker Working Group. Scoring of tumor-infiltrating lymphocytes: From visual estimation to machine learning. Semin Cancer Biol. 2018;52(2):151-7.

16. Wein L, Savas P, Luen SJ, Virassamy B, Salgado R, Loi S. Clinical Validity and Utility of TumorInfiltrating Lymphocytes in Routine Clinical Practice for Breast Cancer Patients: Current and Future Directions. Front Oncol. 2017;7:156.

17. Dieci MV, Radosevic-Robin N, Fineberg S, van den Eynden G, Ternes N, Penault-Llorca F, et al. International Immuno-Oncology Biomarker Working Group on Breast Cancer. Update on tumor-infiltrating lymphocytes (TILs) in breast cancer, including recommendations to assess TILs in residual disease after neoadjuvant therapy and in carcinoma in situ: A report of the International Immuno-Oncology Biomarker Working Group on Breast Cancer. Semin Cancer Biol. 2018;52(2):16-25.

18. Eckstein M, Erben P, Kriegmair MC, Worst TS, Weiß CA, Wirtz RM, et al. Performance of the Food and Drug Administration/EMAapproved programmed cell death ligand-1 assays in urothelial carcinoma with emphasis on therapy stratification for first-line use of atezolizumab and pembrolizumab. Eur J Cancer.
2019;106:234-43.

19. Gravara LD, Battiloro C, Cantile R, Letizia A, Vitiello F, Montesarchio V, et al. Chemotherapy and/or immune checkpoint inhibitors in NSCLC first-line setting: what is the best approach? Lung Cancer Manag. 2020;9(1):LMT22.

20. Mao Y, Qu Q, Zhang Y, Liu J, Chen X, Shen $\mathrm{K}$. The value of tumor infiltrating lymphocytes (TILs) for predicting response to neoadjuvant chemotherapy in breast cancer: a systematic review and meta-analysis. PLoS One. 2014;9(12):e115103.

21. Loi S. Tumor-infiltrating lymphocytes, breast cancer subtypes and therapeutic efficacy. Oncoimmunology. 2013;2(7):e24720.

22. Criscitiello C, Esposito A, Gelao L, Fumagalli L, Locatelli M, Minchella I, et al. Immune approaches to the treatment of breast cancer, around the corner? Breast Cancer Res. 2014;16(1):204.

23. Schmid P, Adams S, Rugo HS, Schneeweiss $\mathrm{A}$, Barrios $\mathrm{CH}$, Iwata $\mathrm{H}$, et al. Atezolizumab and Nab-Paclitaxel in Advanced TripleNegative Breast Cancer. N Engl J Med. 2018;379(22):2108-21.

24. Helal TE, Ibrahim EA, Alloub AI. Immunohistochemical analysis of tumorinfiltrating lymphocytes in breast carcinoma: relation to prognostic variables. Indian J Pathol Microbiol. 2013;56(2):89-93.

25. Shou J, Zhang Z, Lai Y, Chen Z, Huang J. Worse outcome in breast cancer with higher tumor-infiltrating FOXP3+ Tregs : a systematic review and meta-analysis. BMC Cancer. 2016;16(1):687.

26. Gao G, Wang Z, Qu X, Zhang Z. Prognostic value of tumor-infiltrating lymphocytes in patients with triple-negative breast cancer: a systematic review and meta-analysis. BMC Cancer. 2020;20(1):179.

27. Ahn S, Chung YR, Seo AN, Kim M, Woo JW, Park SY. Changes and prognostic values of tumor-infiltrating lymphocyte subsets after primary systemic therapy in breast cancer. PLoS One. 2020;15(5):e0233037.

28. Giraldo NA, Becht E, Vano Y, SautèsFridman C, Fridman WH. The immune response in cancer: from immunology to pathology to immunotherapy. Virchows Arch. 2015;467(2):127-35.

29. Ohtani H, Mori-Shiraishi K, Nakajima M, Ueki H. Defining lymphocyte-predominant breast cancer by the proportion of lymphocyterich stroma and its significance in routine histopathological diagnosis. Pathol Int. 2015;65(12):644-51.

30. García-Teijido P, Cabal ML, Fernández IP, 
Pérez YF. Tumor-Infiltrating Lymphocytes in Triple Negative Breast Cancer: The Future of Immune Targeting. Clin Med Insights Oncol. 2016;10(Suppl 1):31-9.

31. Hudeček J, Voorwerk L, van Seijen M, Nederlof I, de Maaker M, van den Berg J, et al. International Immuno-Oncology Biomarker Working Group. Application of a risk-management framework for integration of stromal tumor-infiltrating lymphocytes in clinical trials. NPJ Breast Cancer. 2020;6:15.

32. Burstein HJ, Curigliano G, Loibl S, Dubsky P, Gnant M, Poortmans P, et al. Estimating the benefits of therapy for early-stage breast cancer: the St. Gallen International Consensus Guidelines for the primary therapy of early breast cancer 2019. Ann Oncol. 2019;30(10):1541-57.

33. WHO classification of tumours editorial board. Breast Tumours. (WHO classification of tumours series, 5th ed., vol. 2) (International Agency for Research on Cancer, Lyon, France, 2019).
34. Miyashita M, Sasano H, Tamaki K, Hirakawa H, Takahashi Y, Nakagawa S, et al. Prognostic significance of tumor-infiltrating CD8+ and FOXP3+ lymphocytes in residual tumors and alterations in these parameters after neoadjuvant chemotherapy in triple-negative breast cancer: a retrospective multicenter study. Breast Cancer Res. 2015;17(1):124.

35. Rakha EA, Martin S, LeeAH, Morgan D, Pharoah PD, Hodi Z, et al. The prognostic significance of lymphovascular invasion in invasive breast carcinoma. Cancer. 2012;118(15):3670-80.

36. Rakha EA, Reis-Filho JS, Baehner F, Dabbs DJ, Decker T, Eusebi V, et al. Breast cancer prognostic classification in the molecular era: the role of histological grade. Breast Cancer Res. 2010;12(4):207.

37. Rakha EA, Ellis IO. Breast Pathology: Problematic Issues. Grading of Invasive Carcinoma. Springer. pp 87-95. 2016. 